\title{
Synthesis and antibacterial activity of new hydrazide-hydrazones derived from Benzocaine
}

\author{
Muhammed İhsan HAN, Güler GÜROL, Temel YILDIRIM, Sadık KALAYCI, Fikrettin ŞAHİN, Ş. Güniz KÜÇÜKGÜZEL
}

\begin{abstract}
A novel series of new eleven benzocaine hydrazide derivatives, $\mathrm{N}$-(4-\{[2-(nonsubstituted/ substitutedfuryl/ phenyl/ pyridinyl/ thienyl/ pyrrole)methylidene]hydrazinyl] carbonyl $\}$ phenyl) benzamides [3a-k] have been synthesized in this study. The structures of the new compounds were determined by spectral (FT-IR and ${ }^{1} \mathrm{H}-\mathrm{NMR}$ ) methods and their purity was proven by elemental analysis and thin layer chromatography. These
\end{abstract}

compounds were evaluated for in vitro antibacterial activity by using micro-well dilution method against Escherichia coli ATCC 10536, Escherichia coli ATCC 15442, Staphylococcus aureus ATCC 6538, Pseudomonas aeruginosa ATCC 15442, Acinetobacter baumannii, Klebsiella pneumonia ATCC 13883.

Key words: Antibacterial activity; Benzocaine; hydrazidehydrazone; azomethine.
Muhammed İhsan Han, Güler Gürol, Temel Yıldırım, Ş. Güniz Küçükgüzel Department of Pharmaceutical Chemistry, Faculty of Pharmacy, Marmara University, Haydarpaşa 34668 İstanbul, Turkey

Sadık Kalaycı, Fikrettin Şahin

Department of Genetics and Bioengineering, Faculty of Engineering and Architecture, Yeditepe University, Kayışdağı, İstanbul, Turkey

Corresponding Author:

Ş. Güniz Küçükgüzel

e-mail: gkucukguzel@marmara.edu.tr

Submitted / Gönderilme: 03.07.2017 Revised / Düzeltme: 21.08.2017 Accepted / Kabul: $\quad 22.08 .2017$

How to cite this article : Han MI, Gürol G, Yıldırım T, Kalaycı S, Şahin F, Küçükgüzel ŞG. Synthesis and antibacterial activity of new hydrazidehydrazones derived from benzocaine. Marmara Pharm J.2017;21 (4):961-966

\section{Introduction}

In recent years, many species of bacteria have improved resistance as a consequence of erroneous and unnecessary use of antibiotics. Lack of new drug molecules and synthesis of molecules with significant effects canalized researchers to develop new molecules in this area. Previously synthesized molecules with hydrazide-hydrazone structure have been found to have antibacterial activity of a significant level. Benzocaine (ethyl 4-amino-benzoate) is a local anesthetic drug which contains esther funcionality. Hydrazidehydrazones have diverse biological activities [1-12].

In this study, benzocaine hydrazide-hydrazones have been synthesized. The purity of the synthesized compounds have been proven by elementel analysis and melting point assay. Their structure elucidation have been characterized by ${ }^{1} \mathrm{H}-\mathrm{NMR}$ and FT-IR spectroscopic methods. The antibacterial activity of the synthesized compounds were studied against Gram positive and Gram negative bacteria, Escherichia coli, Staphylococcus aureus, Pseudomonas aeruginosa, Acinetobacter baumannii, Klebsiella pneumonia. 


\section{Results and Discussion}

\section{1. Chemistry}

Ethyl 4-amino benzoate (Benzocaine) was used as the starting compound to design several novel hydrazidehydrazones. Firstly p-(benzoylamino)benzoic acid hydrazide was prepared. That compound $\mathbf{1}$ was prepared by benzocaine which was solved in diethylether and benzoyl chloride in cold medium. The reaction of compound 1 with hydrazinehydrate in ethanol resulted in $\mathrm{N}$-[4-(hydrazinylcarbonyl) phenyl]benzamide. Compound $\mathbf{2}$ was condensed with substituted aldehydes in ethanolic medium in the presence of a few drops of glacial acetic acid with refluxed to obtain new $N$-(4-\{[2-(nonsubstituted/substituted furyl/phenyl/ pyridinyl/thienyl/pyrrole)methylidene]hydrazinyl]carbonyl\} phenyl)benzamides (3a-k) (Scheme 1).

The structures of compounds $\mathbf{3 a - k}$ were confirmed by elemental analyses and spectral techniques such as FT-IR and ${ }^{1} \mathrm{H}-\mathrm{NMR}$.

FT-IR spectral data of our novel hydrazide-hydrazones 3a-k were observed amide $\mathrm{N}-\mathrm{H}$, hydrazone $\mathrm{N}-\mathrm{H}$ amide $\mathrm{C}=\mathrm{O}$, hydrazone $\mathrm{C}=\mathrm{O}$ and $\mathrm{C}=\mathrm{N}$ streching data between 3360 $3269,3198-3045,1687-1680,1653-1643$ and $1620-1600 \mathrm{~cm}^{-}$ 1 , respectively.

${ }^{1} \mathrm{H}-\mathrm{NMR}$ spectral data of compounds $\mathbf{3 a - k}$ revealed supporting evidence to identify their structures. The singlet signals belonging to azomethine proton in compounds $3 \mathbf{a}-\mathbf{k}$ were detected at 8.39-8.93 ppm respectively. The chemical shift of the azomethine proton in compounds $3 \mathrm{c}$ were detected in the range of 8.17 and $8.40 \mathrm{ppm}$ as two singlet peaks. The $\mathrm{NH}$-proton of acylhydrazone moiety of compounds $3 \mathbf{a}-\mathbf{k}$ was detected in the range of 10.51-10.56 ppm. In the ${ }^{1} \mathrm{H}-\mathrm{NMR}$ spectra of compounds 3a-k the $\mathrm{NH}$-proton of benzamide was detected in the range of 11.48-12.24 ppm.

In the ${ }^{1} \mathrm{H}$-NMR spectra of compounds $\mathbf{3 d}$, characteristic signals for $\mathrm{CH}_{3}$ moiety have been detected 3.38 ppm as signal proton. The $-\mathrm{CH}_{2}-\mathrm{CH}_{3}$ protons of compound $\mathbf{3 f}$ was detected for $\mathrm{CH}_{3}$ moiety at $1.26 \mathrm{ppm}$ as triplet and for $\mathrm{CH}_{2} 2.83 \mathrm{ppm}$ as quartet signals. In the ${ }^{1} \mathrm{H}-\mathrm{NMR}$ spectra of compounds $\mathbf{3 k}$ which were derived from piperidine aldehyde, characteristic signals for $\mathrm{CH}$ moiety were detected in the range of 1.58-3.27 ppm.

\subsection{Biological Activity}

The antimicrobial activities of all compounds were evaluated in the Department of Genetics and Bioengineering, Faculty of Engineering, Yeditepe University. The sensitivity of the bacterial strains towards the compounds was evaluated from the minimal inhibitory concentration (MIC) values obtained by the micro-well dilution method. The antibacterial activity of the compounds were evaluated against 5 bacterial cultures. The microorganisms used were Escherichia coli ATCC 10536, Escherichia coli ATCC 15442, Staphylococcus aureus ATCC 6538, Pseudomonas aeruginosa ATCC 15442, Acinetobacter baumannii, Klebsiella pneumonia ATCC 13883. The results of antibacterial activity of synthesized compounds $\mathbf{3} \mathbf{a}-\mathbf{k}$ are given Table 1.

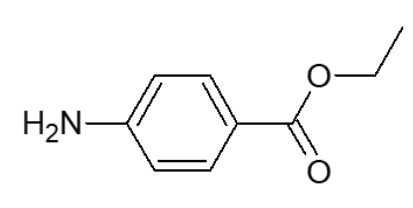

Benzocaine

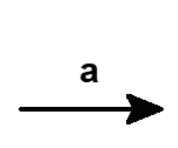

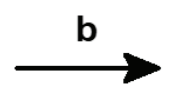

1

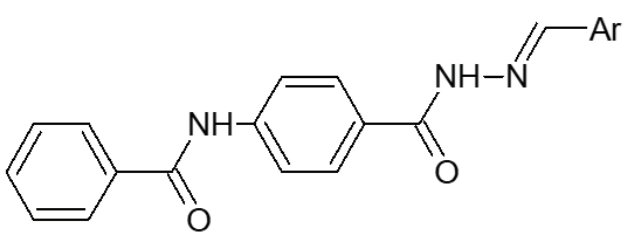

3a-k

Scheme 1. Synthetic route to benzocaine hydrazide-hydrazones (3a-k). a) $\left.\left.\mathrm{C}_{6} \mathrm{H}_{5} \mathrm{COCl}, \mathrm{Et}_{2} \mathrm{O} ; \mathbf{b}\right) \mathrm{NH}_{2} \mathrm{NH}_{2} . \mathrm{H}_{2} \mathrm{O}, \mathrm{EtOH} \mathbf{c}\right) \mathrm{Ar}-$ $\mathrm{CHO}, \mathrm{EtOH}$. 
Table 1. Antibacterial activity results of the synthesized compounds $\mathbf{3 a - k}$

\begin{tabular}{|c|c|c|c|c|c|}
\hline \multirow[b]{2}{*}{$\begin{array}{l}\text { COMPOUND ID } \\
\text { (LAB CODE) }\end{array}$} & \multicolumn{5}{|c|}{ Antibacterial activity $(\mathrm{MIC}, \mu \mathrm{g} / \mu \mathrm{l})$} \\
\hline & $\begin{array}{c}\text { Escherichia coli ATCC } \\
\text { 10536-ATCC } 15442\end{array}$ & $\begin{array}{c}\text { Staphylococcus aureus } \\
\text { ATCC } 6538\end{array}$ & $\begin{array}{c}\text { Pseudomonas } \\
\text { aeruginosa ATCC } \\
15442\end{array}$ & $\begin{array}{c}\text { Acinetobacter } \\
\text { baumannii }\end{array}$ & $\begin{array}{c}\text { Klebsiella pneumoniae } \\
\text { ATCC } 13883\end{array}$ \\
\hline 3a (SGK 581) & 8.192 & 4.096 & $>8.192$ & $>8.192$ & 8.192 \\
\hline 3b (SGK 582) & 8.192 & 2.048 & 4.096 & $>8.192$ & 8.192 \\
\hline $3 c$ (SGK 583) & 8.192 & 8.192 & 4.096 & $>8.192$ & 8.192 \\
\hline 3d (SGK 584) & 4.096 & 2.048 & 2.048 & 8.192 & 4.096 \\
\hline 3e (SGK 585) & 4.096 & 2.048 & 2.048 & 8.192 & 4.096 \\
\hline 3f (SGK 587) & 2.048 & 2.048 & 2.048 & 4.096 & 4.096 \\
\hline 3g (SGK 588) & 2.048 & 2.048 & 2.048 & 8.192 & 4.096 \\
\hline 3h (SGK 589) & $>8.192$ & $>8.192$ & $>8.192$ & $>8.192$ & $>8.192$ \\
\hline $3 \mathrm{i}($ SGK 590) & $>8.192$ & $>8.192$ & $>8.192$ & $>8.192$ & $>8.192$ \\
\hline $3 \mathrm{j}$ (SGK 591) & $>8.192$ & $>8.192$ & $>8.192$ & $>8.192$ & $>8.192$ \\
\hline 3k (SGK 592) & $>8.192$ & $>8.192$ & $>8.192$ & $>8.192$ & $>8.192$ \\
\hline
\end{tabular}

\section{Conclusion}

Through condensation of $\mathrm{N}$-[4-(hydrazinylcarbonyl) phenyl]benzamide (2) and selected aldehydes, 11 new acylhydrazone derivatives were synthesized and evaluated for their antibacterial activity. None of the compounds tested (compounds $\mathbf{3 a - k}$ ) were found to be active against both Gram (+) and Gram (-) bacterial strains.

\section{Materials and Methods}

Benzocaine was liberally ensured Merck. All aldehydes were purchased from Fluka and Aldrich. All other chemicals were purchased from Merck. Melting points were taken on Schmelzpunktbestimmer 9300 SMP II apparatus and are uncorrected. Synthesis of these compounds were carried out in Memmert WNB14 instrument and Heidolph MR Plug radley. Elemental analyses were performed on VarioMICRO V1.5.7.* instrument. FT-IR spectra were run on Schimadzu FTIR-8400S spectrophotometer. ${ }^{1} \mathrm{H}-\mathrm{NMR}$ spectra were obtained on a BRUKER AVANCE-DPX $400^{*}$ instrument (*This instruments exist in İnönü University Scientific and Technological Research Center).

\subsection{Chemistry}

Preparation of ethyl 4-[(phenylcarbonyl)amino]benzoate (1) and $N$-[4-(hydrazinylcarbonyl) phenyl]benzamide (2)

Benzocaine (0.05 mol, $8.25 \mathrm{~g}$ ) was dissolved in diethylether $(50 \mathrm{~mL})$. Simultaneously solution of benzoylchloride $(0.05$ mol, $6 \mathrm{~mL}$ ) was added dropwise to that liquid with stirring. This reaction becomes in cold atmosphere and it cooled with ice bath (approximately 5-10 ${ }^{\circ} \mathrm{C}$ ). Almost the mixture was stirred 45 minutes. The solid which was fell down filtered and washed with cold water. Extra benzoyl chloride go away from the solid with water. The compound (1) was crystallized from ethanol (M.p. $139{ }^{\circ} \mathrm{C}$, Lit. M.p. $140^{\circ} \mathrm{C}[13]$ ).

Compound 1 (0.04 mol,10.77g) and hydrazine-hydrate (\%80, $7,5 \mathrm{ml}$ ) was heated under the reflux in $50 \mathrm{~mL}$ ethanol for 2 hours. The mixture was cooled at room temperature after stopped the reaction. The precipitate was filtered and washed with water. The yield crystallized from ethanol (M.p $237^{\circ} \mathrm{C}$, Lit. M.p. $\left.240{ }^{\circ} \mathrm{C}[13]\right)$.

\section{General procedure for the synthesis of arylhydrazones of} $\mathrm{N}$-[4-(hydrazinylcarbonyl) phenyl]benzamide (3a-k)

A solution of compound $2(0.001 \mathrm{~mol}, 0.255 \mathrm{~g})$ in $20 \mathrm{~mL}$ ethanol and appropriate aldehyde $(0.001 \mathrm{~mol})$ were heated $\left(100{ }^{\circ} \mathrm{C}\right)$ under reflux for 8 hours. After the mixture was cooled at room temperature and ethanol was evaporated. The product was dried and crystallized with ethanol.

\section{$N$-(4-\{[2-(4-bromothiophene-2-yl)methylidene] hydrazinyl $]$ carbonyl $\}$ phenyl)benzamide (3a)}

Yield \%81; M.p. $294{ }^{\circ} \mathrm{C}$; Rf 0.67; FT-IR v ${ }_{\max }\left(\mathrm{cm}^{-1}\right): 3360$ (amide NH str.), 3198 (hydrazone NH str.), 3003 (arom. CH str.), 1680 (amide CO str.), 1645 (hydrazone CO str.), 1606 (hydrazone $\mathrm{C}=\mathrm{N}$ str.), 1593, 1541, 1500, 1489 (aromatic C=C str., amide $\mathrm{CN}$ str., amide and hydrazone $\mathrm{NH}$ bending), 1058 (arom. C-Br str.); ${ }^{1} \mathrm{H}-\mathrm{NMR}$ (DMSO-d, $\left.300 \mathrm{MHz}\right) \delta$ (ppm): 7.53-7.99 (11H, m, Ar-H), $8.63(1 \mathrm{H}, \mathrm{s},-\mathrm{N}=\mathrm{CH}), 10.53$ (1H, s, CO-NH), $11.90(1 \mathrm{H}, \mathrm{s}, \mathrm{CO}-\mathrm{NH}-\mathrm{N})$. Anal. Calcd for $\mathrm{C}_{19} \mathrm{H}_{14} \mathrm{BrN}_{3} \mathrm{O}_{2} \mathrm{~S}$ : C, 53.28; H, 3.29; N, 9.81; S, 7.49. Found: C, 53.97; H, 3.72; N, 9.94; S, 7.40. 
$\mathrm{N}$-(4-\{[2-(5-nitrofuran-2-yl) methylidene]hydrazinyl] carbonyl $\}$ phenyl)benzamide ( $3 b$ )

Yield \%83; M.p. 307-308 ${ }^{\circ}$; Rf 0.38; FT-IR v ${ }_{\max }\left(\mathrm{cm}^{-1}\right)$ : 3321 (amide NH str.), 3144 (hydrazone NH str.), 3003 (arom. CH str.), 1687 (amide CO str.), 1651 (hydrazone CO str.), 1612 (hydrazone CN str.), 1599, 1541, 1508, 1469, 1402 (aromatic $\mathrm{C}=\mathrm{C}$ str., amide $\mathrm{CN}$ str., nitro $\mathrm{N}=\mathrm{O}$ asm. str., amide and hydrazone $\mathrm{NH}$ bending), 1346 (nitro $\mathrm{N}=\mathrm{O}$ sym str.) ; ${ }^{1} \mathrm{H}-\mathrm{NMR}$ (DMSO-d, $\left.300 \mathrm{MHz}\right) \delta(\mathrm{ppm}): 7.26-7.99(11 \mathrm{H}, \mathrm{m}$, Ar-H), $8.40(1 \mathrm{H}, \mathrm{s},-\mathrm{N}=\mathrm{CH}), 10.56(1 \mathrm{H}, \mathrm{s}, \mathrm{CO}-\mathrm{NH}), 12.21$ $(1 \mathrm{H}, \mathrm{s}, \mathrm{CO}-\mathrm{NH}-\mathrm{N})$. Anal. Calcd for $\mathrm{C}_{19} \mathrm{H}_{14} \mathrm{~N}_{4} \mathrm{O}_{5}: \mathrm{C}, 60.32 ; \mathrm{H}$, 3.73; N, 14.81. Found: C, 60.09; H, 3.65; N, 14.59.

\section{$N$-(4-\{[2-(furan-3-yl) methylidene]hydrazinyl $]$ carbonyl $\}$} phenyl)benzamide (3c)

Yield \%62; M.p. 308-309 ${ }^{\circ} \mathrm{C}$; Rf 0.43 FT-IR v ${ }_{\max }\left(\mathrm{cm}^{-1}\right): 3335$ (amide NH str.), 3184 (hydrazone NH str.), 3003 (arom. CH str.), 1680 (amide CO str.), 1643 (hydrazone CO str.), 1600 (hydrazone CN str.), 1570, 1527, 1508, 1489 (aromatic C=C str., amide $\mathrm{CN}$ str., amide and hydrazone $\mathrm{NH}$ bending); ${ }^{1} \mathrm{H}-\mathrm{NMR}$ (DMSO-d, $\left.300 \mathrm{MHz}\right) \delta$ (ppm): 6.82-7.99 $(12 \mathrm{H}$, m, Ar-H), 8.17, $8.40(1 \mathrm{H}, \mathrm{s},-\mathrm{N}=\mathrm{CH}), 10.52(1 \mathrm{H}, \mathrm{s}, \mathrm{CO}-\mathrm{NH})$, $11.67(1 \mathrm{H}, \mathrm{s}, \mathrm{CO}-\mathrm{NH}-\mathrm{N})$. Anal. Calcd for $\mathrm{C}_{19} \mathrm{H}_{15} \mathrm{~N}_{3} \mathrm{O}_{3}: \mathrm{C}$, 68.46; H, 4.54; N, 12.61. Found: C, 67.77; H, 4.48; N, 12.44.

\section{$N$-(4-\{[2-(1-methyl-1H-pyrrole-2-yl)methylidene] hydrazinyl] carbonyl $\}$ phenyl)benzamide (3d)}

Yield \%63; M.p. 254-255 ${ }^{\circ} \mathrm{C}$; Rf 0.64; IR $\mathrm{v}_{\max }\left(\mathrm{cm}^{-1}\right): 3292$ (amide NH str.), 3080 (hydrazone NH str.), 3003 (arom. CH str.), 2933 (alif. CH str.), 1680 (amide CO str.), 1651 (hydrazone CO str.), 1620 (hydrazone CN str.), 1602, 1543, 1506, 1489 (aromatic $\mathrm{C}=\mathrm{C}$, amide $\mathrm{CN}$ str., amide and hydrazone NH bending); ${ }^{1} \mathrm{H}-\mathrm{NMR}$ (DMSO- $\mathrm{d}_{6}, 300 \mathrm{MHz}$ ) $\delta$ (ppm): $3.88\left(3 \mathrm{H}, \mathrm{s}, \mathrm{Ar}-\mathrm{CH}_{3}\right)$ 6.10-7.99 $(12 \mathrm{H}, \mathrm{m}, \mathrm{Ar}-\mathrm{H})$, $8.38(1 \mathrm{H}, \mathrm{s},-\mathrm{N}=\mathrm{CH}), 10.51(1 \mathrm{H}, \mathrm{s}, \mathrm{CO}-\mathrm{NH}), 11.48(1 \mathrm{H}, \mathrm{s}$, CO-NH-N). Anal. Calcd for $\mathrm{C}_{20} \mathrm{H}_{18} \mathrm{BrN}_{4} \mathrm{O}_{2} \cdot 1 / 2 \mathrm{C}_{2} \mathrm{H}_{5} \mathrm{OH}$ : C, 68.22; H, 5.68; N, 15.16. Found: C, 68.16; H, 4.86; N, 15.96.

\section{$N$-(4-\{[2-(2,6-difluorobenzylidene)hydrazinyl $]$ carbonyl $\}$ phenyl)benzamide (3e)}

Yield \%90; M.p. $283-285^{\circ} \mathrm{C}$; Rf 0.50; FT-IR v ${ }_{\max }\left(\mathrm{cm}^{-1}\right): 3271$ (amide NH str.), 3045 (hydrazone NH str.), 3001 (arom. CH str.), 1689 (amide CO str.), 1645 (hydrazone CO str.), 1602 (hydrazone CN str.), 1591, 1545, 1510, 1489 (aromatic C=C str., amide CN str., amide and hydrazone $\mathrm{NH}$ bending), 1122 (arom C-F str.); ${ }^{1} \mathrm{H}-\mathrm{NMR}$ (DMSO-d $\left.{ }_{6}, 300 \mathrm{MHz}\right) \delta$ (ppm): 7.24-7.99 (12H, m, Ar-H), $8.63(1 \mathrm{H}, \mathrm{s},-\mathrm{N}=\mathrm{CH}), 10.54$ (1H, s, CO-NH), $11.94(1 \mathrm{H}, \mathrm{s}, \mathrm{CO}-\mathrm{NH}-\mathrm{N})$. Anal. Calcd for $\mathrm{C}_{21} \mathrm{H}_{15} \mathrm{~F}_{2} \mathrm{~N}_{3} \mathrm{O}_{2}$ : C, 66.49; H, 3.99; N, 11.08. Found: C, 67.30; $\mathrm{H}, 4.14 ; \mathrm{N}, 10.82$.

$N$ - [4-( \{2-[(5-ethylthiophene-2-yl)methylidene] hydrazinyl $\}$ carbonyl)phenyl]benzamide (3f)

Yield \%36; M.p. $237^{\circ} \mathrm{C}$; Rf 0.55; FT-IR $\mathrm{v}_{\max }\left(\mathrm{cm}^{-1}\right): 3335$ (amide NH str.), 3157 (hydrazone NH str.), 3003 (arom. CH str.), 2933 (aliphatic CH str.), 1680 (amide CO str.), 1653 (hydrazone CO str.), 1604 (hydrazone CN str.), 1587, 1556, 1502, 1487 (aromatic $\mathrm{C}=\mathrm{C}$ str., amide $\mathrm{CN}$ str., amide and hydrazone $\mathrm{NH}$ bending); ${ }^{1} \mathrm{H}-\mathrm{NMR}$ (DMSO- $\mathrm{d}_{6}, 300 \mathrm{MHz}$ ) $\delta(\mathrm{ppm}): 1.26\left(3 \mathrm{H}, \mathrm{t},-\mathrm{CH}_{2}-\mathrm{CH}_{3}\right) 2.83\left(2 \mathrm{H}, \mathrm{q},-\mathrm{CH}_{2}-\mathrm{CH}_{3}\right)$ 6.87-7.98 $(11 \mathrm{H}, \mathrm{m}, \operatorname{Ar}-\mathrm{H}), 8.57(1 \mathrm{H}, \mathrm{s},-\mathrm{N}=\mathrm{CH}), 10.52$ $(1 \mathrm{H}, \mathrm{s}, \mathrm{CO}-\mathrm{NH}), 11.69(1 \mathrm{H}, \mathrm{s}, \mathrm{CO}-\mathrm{NH}-\mathrm{N})$. Anal. Calcd for $\mathrm{C}_{21} \mathrm{H}_{19} \mathrm{~N}_{3} \mathrm{O}_{2} \mathrm{~S}$ : C, 66.82; H, 5.07; N, 11.13; $\mathrm{S}, 8.49$. Found: C, $66.44 ; \mathrm{H}, 4.93 ; \mathrm{N}, 10.92 ; \mathrm{S}, 8.08$.

\section{$\mathrm{N}$-[4-(\{2-[4-(trifluoromethoxy)benzylidene]hydrazinyl $\}$} carbonyl)phenyl]benzamide (3g)

Yield \%77; M.p. $31{ }^{\circ} \mathrm{C}$; Rf 0.55; FT-IR $\mathrm{v}_{\max }\left(\mathrm{cm}^{-1}\right)$ : 3327 (amide NH str.), 3159 (hydrazone NH str.), 2997 (arom. CH str.), 1680 (amide CO str.), 1653 (hydrazone CO str.), 1606 (hydrazone CN str.) 1581, 1543, 1506, 1489 (aromatic C=C str., amide $\mathrm{CN}$ str., amide and hydrazone $\mathrm{NH}$ bending), 1151 (ether CO str.); ${ }^{1} \mathrm{H}-\mathrm{NMR}$ (DMSO-d $\left.{ }_{6}, 300 \mathrm{MHz}\right) \delta$ (ppm): 7.53-8.00 (13H, m, Ar-H), $8.49(1 \mathrm{H}, \mathrm{s},-\mathrm{N}=\mathrm{CH}), 10.54$ $(1 \mathrm{H}, \mathrm{s}, \mathrm{CO}-\mathrm{NH}), 11.91(1 \mathrm{H}, \mathrm{s}, \mathrm{CO}-\mathrm{NH}-\mathrm{N})$. Anal. Calcd for $\mathrm{C}_{22} \mathrm{H}_{16} \mathrm{~F}_{3} \mathrm{~N}_{3} \mathrm{O}_{3}: \mathrm{C}, 61.83 ; \mathrm{H}, 3.77 ; \mathrm{N}, 9.83$. Found: $\mathrm{C}, 61.00 ; \mathrm{H}$, $3.62 ; \mathrm{N}, 9.74$.

\section{$N$-(4-\{[2-(4-cyanobenzylidene)hydrazinyl $]$ carbonyl $\}$ phenyl)benzamide (3h)}

Yield \%88; M.p. 303-304 ${ }^{\circ} \mathrm{C}$; Rf 0.51; FT-IR v max $\left(\mathrm{cm}^{-1}\right): 3323$ (amide NH str.), 3126 (hydrazone NH str.), 3022 (arom. CH str.), 2225 (cyano CN str.) 1680 (amide CO str.), 1653 (hydrazone CO str.), 1606 (hydrazone CN str.), 1589, 1541, 1502, 1487 (aromatic $\mathrm{C}=\mathrm{C}$ str., amide $\mathrm{CN}$ str., amide and hydrazone NH bending); ${ }^{1} \mathrm{H}-\mathrm{NMR}$ (DMSO- $\left.\mathrm{d}_{6}, 300 \mathrm{MHz}\right) \delta$ (ppm): 7.53-7.99 (13H, m, Ar-H), $8.51(1 \mathrm{H}, \mathrm{s},-\mathrm{N}=\mathrm{CH}), 10.55$ (1H, s, CO-NH), $12.05(1 \mathrm{H}, \mathrm{s}, \mathrm{CO}-\mathrm{NH}-\mathrm{N})$. Anal. Calcd for $\mathrm{C}_{22} \mathrm{H}_{16} \mathrm{~N}_{4} \mathrm{O}_{2} .1 / 2 \mathrm{C}_{2} \mathrm{H}_{5} \mathrm{OH}: \mathrm{C}, 70.51 ; \mathrm{H}, 4.85 ; \mathrm{N}, 14.30$. Found: C, 70.49; H, 4.40; N, 14.82 . 
$N$ - [ 4- ( $\{2-[3,5-b i s($ trifluoromethyl) benzylidene $]$ hydrazinyl\}carbonyl)phenyl]benzamide (3i)

Yield \%88; M.p. $297{ }^{\circ} \mathrm{C}$; Rf 0.55; FT-IR $\mathrm{v}_{\max }\left(\mathrm{cm}^{-1}\right): 3298$ (amide NH str.), 3124 (hydrazone NH str.), 3030 (arom. CH str.), 1680 (amide CO str.), 1641 (hydrazone CO str.), 1610 (hydrazone CN str.), 1600, 1533, 1508, 1487 (aromatic C=C str., amide $\mathrm{CN}$ str., amide and hydrazone $\mathrm{NH}$ bending); ${ }^{1} \mathrm{H}-\mathrm{NMR}$ (DMSO-d $\left.\mathrm{d}_{6}, 300 \mathrm{MHz}\right) \delta(\mathrm{ppm}): 7.54-8.61(12 \mathrm{H}, \mathrm{m}$, Ar-H, 1H, s, -N=CH), $10.56(1 \mathrm{H}, \mathrm{s}, \mathrm{CO}-\mathrm{NH}), 12.24(1 \mathrm{H}, \mathrm{s}$, CO-NH-N). Anal. Calcd for $\mathrm{C}_{23} \mathrm{H}_{15} \mathrm{~F}_{6} \mathrm{~N}_{3} \mathrm{O}_{2}: \mathrm{C}, 57.63 ; \mathrm{H}, 3.15$; N, 8.77. Found: C, 57.52; H, 3.12; N, 8.63.

\section{$N$-[4-(\{2-[(5-(2-nitrophenyl)furan-2-yl)methylidene] hydrazinyl\}carbonyl)phenyl] benzamide ( $3 \mathbf{j})$}

Yield \%91; M.p. 265-266 ${ }^{\circ} \mathrm{C}$; Rf 0.69; FT-IR v ${ }_{\max }\left(\mathrm{cm}^{-1}\right): 3327$ (amide NH str.), 3134 (hydrazone NH str.), 2997 (arom. CH str.), 1683 (amide CO str.), 1654 (hydrazone CO str.), 1608 (hydrazone CN str.), 1591, 1545, 1508, 1489, 1404 (aromatic $\mathrm{C}=\mathrm{C}$ str., nitro $\mathrm{NO}_{2}$ asm. str., amide $\mathrm{CN}$ str., amide and hydrazone $\mathrm{NH}$ bending) 1404 (nitro $\mathrm{NO}_{2}$ sym. str.); ${ }^{1} \mathrm{H}-\mathrm{NMR}$ (DMSO-d, $300 \mathrm{MHz}) \delta(\mathrm{ppm}): 7.10-7.99(15 \mathrm{H}, \mathrm{m}, \mathrm{Ar}-\mathrm{H})$, $8.39(1 \mathrm{H}, \mathrm{s},-\mathrm{N}=\mathrm{CH}), 10.54(1 \mathrm{H}, \mathrm{s}, \mathrm{CO}-\mathrm{NH}), 11.83(1 \mathrm{H}, \mathrm{s}$, CO-NH-N). Anal. Calcd for $\mathrm{C}_{25} \mathrm{H}_{18} \mathrm{~N}_{4} \mathrm{O}_{3}$ : C, 66.08; H, 3.99; $\mathrm{N}, 12.33$. Found: C, 65.84; H, 3.91; N, 12.14 .

\section{$N$-(4-\{[2-(4-(piperidine-1-yl)benzylidene)hydrazinyl] carbonyl $\}$ phenyl)benzamide (3k)}

Yield \%75; M.p. $299^{\circ} \mathrm{C}$; Rf 0.48; FT-IR $\mathrm{v}_{\max }\left(\mathrm{cm}^{-1}\right): 3317$ (amide NH str.), 3163 (hydrazone NH str.), 3034 (arom. CH str.), 1680 (amide CO str.), 1653 (hydrazone CO str.), 1604 (hydrazone CN str.), 1597, 1543, 1506, 1489 (aromatic C=C str., amide $\mathrm{CN}$ str., amide and hydrazone $\mathrm{NH}$ bending); ${ }^{1} \mathrm{H}-\mathrm{NMR}$ (DMSO-d $\left.\mathrm{d}_{6} 300 \mathrm{MHz}\right) \delta(\mathrm{ppm}): 1.58(5 \mathrm{H}, \mathrm{s}$, piperidine $\left.-\mathrm{CH}_{2}\right), 3.27\left(5 \mathrm{H}, \mathrm{s}\right.$, piperidine $\left.-\mathrm{CH}_{2}\right)$ 6.96-8.32 $(15 \mathrm{H}, \mathrm{m}, \mathrm{Ar}-\mathrm{H} ; 1 \mathrm{H}, \mathrm{s},-\mathrm{N}=\mathrm{CH}), 10.51(1 \mathrm{H}, \mathrm{s}, \mathrm{CO}-\mathrm{NH}), 11.55$ $(1 \mathrm{H}, \mathrm{s}, \mathrm{CO}-\mathrm{NH}-\mathrm{N})$. Anal. Calcd for $\mathrm{C}_{26} \mathrm{H}_{26} \mathrm{~N}_{4} \mathrm{O}_{2}$ : C, 71.64; H, 6.19; N, 12.85. Found: C, 71.38; H, 5.92; N, 12.52.

\subsection{Antibacterial activity}

All synthesized compounds were evaluated for antibacterial activity. Activity experiments were carried out in Yeditepe University, Faculty of Engineering, Department of Genetic and Bioengineering. Gram positive and Gram negative bacteria, Escherichia coli ATCC 10536, Escherichia coli ATCC 15442, Staphylococcus aureus ATCC 6538, Pseudomonas aeruginosa
ATCC 15442, Acinetobacter baumannii, Klebsiella pneumonia ATCC 13883 were used in activity studies. Antibacterial activities of the compounds tested against that bacteria species based on micro-well dilution assay. The sensitivity of the bacterial strains towards the compounds was quantitively evaluated from the minimal inhibitory concentration (MIC) values obtained by the micro-well dilution method. The inocula of the bacterial strains were prepared from $12 \mathrm{~h}$ broth cultures and suspensions were adjusted to $0.5 \mathrm{McFarland}$ standard turbidity. Compounds dissolved in DMSO were first prepared at the highest concentration to be tested (200 $\mu \mathrm{g} / \mathrm{mL}$ ), and then serial two-fold dilutions were made in order to obtain a concentration range from 6.25 to $200 \mu \mathrm{g} /$ $\mathrm{mL}$, in $15 \mathrm{~mL}$ sterile test tubes containing nutrient broth. The 96-well plates were prepared by dispensing into each well 95 $\mu \mathrm{L}$ of nutrient broth and $5 \mu \mathrm{L}$ of the inoculum. $200 \mu \mathrm{L}$ of nutrient broth without inoculum was transferred into the first wells as positive control. Aliquots, $(100 \mu \mathrm{L})$ taken from the $200 \mu \mathrm{g} / \mathrm{mL}$ stock solution, were added to the second well. $100 \mu \mathrm{L}$ from the respective serial dilutions was transferred into 5 consecutive wells. The last well containing $195 \mu \mathrm{L}$ of nutrient broth without compound and $5 \mu \mathrm{L}$ of the inoculum on each strip was used as negative control. Contents of each well were mixed on plate shaker at $300 \mathrm{rpm}$ for $20 \mathrm{~s}$ and then incubated at appropriate temperatures for $24 \mathrm{~h}$. Microbial growth in each medium was determined by reading the absorbance (Abs) at $630 \mathrm{~nm}$ using the ELx 800 universal microplate reader (Biotek Instrument inc, Highland Park, Vermont, USA) and confirmed by plating $5 \mu \mathrm{L}$ samples from clear wells on nutrient agar medium. The MIC was defined as the lowest concentration of the compounds to inhibit the growth of microorganisms. Ampisillin was used as the positive sensitivity reference standard for bacteria $[14,15]$.

\section{References}

1. Kömürcü ŞG, Rollas S, Ülgen M, Gorrod JW, Çevikbaş A. Evaluation of some arylhydrazones of p-aminobenzoic acid hydrazide as antimicrobial agents and their in-vitro hepatic microsomal metabolism. Boll Chim Farmaceutico 1995; 134: 375-9.

2. Küçükgüzel ŞG, Oruç EE, Rollas S, Şahin F, Özbek A. Synthesis, characterization and biological activity of novel 4-thiazolidinones, 1,3,4-oxadiazoles and some related compounds. Eur J Med Chem 2002; 37: 197-206.

3. Küçükgüzel ŞG, Mazı A, Şahin F, Öztürk S, Stables J. Synthesis and biological activities of diflunisal hydrazide-hydrazones. Eur J Med Chem 2003; 38:1005-1013.

4. Rollas S, Küçükgüzel ŞG. Biological activities of hydrazone derivatives. Molecules 2007; 12:1910-39. 
5. Çıkla P, Özsavcı D, Özakpınar ÖB, Şener A, Çevik Ö, Turan SÖ, Akbuğa J, Şahin F, Küçükgüzel ŞG. Synthesis, cytotoxicity and pro-apoptosis of etodolac hydrazide derivatives as anticancer agents. Arch Pharm 2013; 346: 367-79.

6. Çıkla P, Tatar E, Küçükgüzel İ, Şahin F, Yurdakul D, Basu A, Krishnan R, Nichols DB, Basu NK, Küçükgüzel ŞG. Synthesis and characterization of flurbiprofen hydrazide derivatives as potential anti-HCV, anticancer, antimicrobial agents. Med Chem Res 2013; 22: 5685-99.

7. Aydın S, Basu NK, Arora P, Basu A, Nichols DB, Talele TT, Akkurt M, Çelik İ, Büyükgüngör O, Küçükgüzel ŞG. Microwave assisted synthesis of some novel flurbiprofen hydrazide-hydrazones as anti-HCV NS5B and anticancer agents. Marmara Pharm J 2013; 17: 26-34.

8. Küçükgüzel ŞG, Koç D, Çıkla P, Özsavcı D, Özakpınar ÖB, Tiber PM, Orun O, Erzincan P, Erdem SS, Şahin F. Synthesis of tolmetin hydrazide-hydrazones and discovery of a potent apoptosis inducer in colon cancer cells. Arch Pharm 2015; 348: 730-42.

9. Şenkardeş S, Basu NK, Durmaz İ, Manvar D, Basu A, Atalay $\mathrm{R}$, Küçükgüzel ŞG. Synthesis of novel diflunisal hydrazidehydrazones as anti-hepatitis $\mathrm{C}$ virus agents and hepatocellular carcinoma inhibitors. Eur J Med Chem 2016; 108: 301-8.
10. Sarıözkan S, Gaffari T, Pelin ÇS, Güvenç M, Yüce A, Yay A, Canturk F, Küçükgüzel ŞG. Effect of etodolac hydrazone, a new compound synthesized from etodolac, on sperm quality, testicular lipid peroxidation, apoptosis and sperm DNA integrity. Andrologia 2016; 48: 177-88.

11. Tatar E, Şenkardes S, Sellitepe HE, Küçükgüzel ŞG, Karaoglu ŞA, Bozdeveci A, De Clercq E, Pannecouque C, Hadda TB, Küçükgüzel İ. Synthesis, and prediction of molecular properties and antimicrobial activity of some acylhydrazones derived from N-(arylsulfonyl)methionine. Turk J Chem 2016; 40: 510-34.

12. Keogh A, Şenkardeş S, Idle JR, Küçükgüzel ŞG, Beyoğlu D. A novel anti-hepatitis $C$ virus and antiproliferative agent alters metabolic networks in HepG2 and Hep3B Cells. Metabolites 2017; 7: 2-16.

13. Durgun BB, Rollas S. Synthesis and characterization of some arylhydrazones of p-(benzoylamino) benzoic acid hydrazide. J Pharm Univ Marm 1991; 7: 107-11.

14. Kalaycı S, Iyigündoğdu ZU, Yazıcı MM, Asutay BA, Demir O, Şahin F. Evaluation of antimicrobial and antiviral activities of different venoms. Infect Disord-Drug Targets 2016; 16: 44-53.

15. Kalaycı S, Demirci S, Şahin F. Antimicrobial properties of various psychotropic drugs against broad range microorganisms. Curr Psychopharmacol 2014; 3: 195-202. 